\title{
Trace-Orthonormal Full-Diversity Cyclotomic Linear Dispersion Codes Using Coordinate Interleaving
}

\author{
Wen Mi, and Wen Chen \\ Department of Electronic Engineering, \\ Shanghai Jiao Tong University, \\ Shanghai, China. 200240. \\ Email: \{miwen,wenchen\}@ sjtu.edu.cn
}

\begin{abstract}
In this paper, we improve the Trace-orthonormal full-diversity cyclotomic space-time codes using coordinate interleaving. We find that in the original design of full-rate fulldiversity cyclotomic linear dispersion codes (FR-FD LDC), the constellation is restricted. Fortunately, a coordinate interleaving method enables not only symbol-level diversity but also coordinate-level diversity for high rate $L D$ code design. Using this method, we can improve the performance of the cyclotomic LD codes over the constellations without the constellation restriction, in block and fast fading channels.
\end{abstract}

Index Terms-MIMO communication systems, linear dispersion codes, full diversity, full rate, coordinate interleaving.

\section{INTRODUCTION}

Multiple-input multiple-output wireless links are important recent developments to meet the challenges brought along by the rapidly growing demands in wireless communications. The importance of the MIMO communication systems lies in the fact that $M$ transmitter antennas and $N$ receiver antennas are used enabling the system to exploit the high performance provided by the space diversity available in MIMO channels [1], [2]. Full diversity (FD) is achieved when the total degrees of freedom $(M \times N)$ offered in the multiantenna system are utilized. This will ensure a good performance in terms of probability of error for detecting the transmitted symbols at a high signal-to-noise ratio (SNR) . A full symbol rate, on the other hand, is achieved when one symbol is transmitted by each of the multiple transmitter antennas per time slot (often called a "channel use"). Specifically, for $M$ transmitter antennas, we will have $M$ symbols per channel use at full rate (FR).

To achieve good performance and good transmission rate in MIMO communications, Hassibi [3] proposed linear dispersion (LD) codes in which the transmitted codeword is a linear combination of certain weighted matrices. The key to LD code design is that the basic matrices are optimized such that the resulting codes maximize the ergodic capacity of the MIMO system. But, for the LD codes proposed in [3], good error probability performance is not guaranteed. Then Heath [4] proposed an LD code design using frame theory that typically performs well in terms of both ergodic capacity and error performance. However, their design still cannot guarantee full diversity.

Investigation based on number theory has shown that it is possible to design FR-FD LD codes. In [5], the authors examined the design of LD codes applied to a MIMO communication system from both the information-theoretic and detection error viewpoints. By applying cyclotomic field theory, this has led us to reach a very efficient design of full diversity rectangular LD code at a symbol transmission rate equal to the number of the transmitter antennas. But this design is only adapt to some restricted constellation. In [6], the authors proposed a general coordinate-interleaving method for LD codes, which enables not only symbol-level diversity but also coordinate-level diversity for high rate LD codes. In this paper, we propose trace-orthonormal full-diversity cyclotomic LD codes using coordinate interleaving to impove the diversity performance over some constellations in block and rapid fading channels.

\section{MIMO SySTEM WITH LD CODES}

We consider a MIMO system with $M$ transmit antennas, $N$ receive antennas, and an interval of $T$ symbols available to us during which the propagation channel is constant and known to the receiver. The information signal symbols $\left\{s_{k}\right\}$ are randomly selected from a certain constellation with zero mean and unit variance. At the $t$ th transmission time slot, an $M$-dimensional vector (a coded version of the information symbols) is transmitted. Thus, for $T$ transmission time slots, there are $T$ coded signal vectors forming an $M \times T$ coded signal matrix $S$ given by [3]

$$
\mathbf{S}=\sum_{k=1}^{K}\left(\mathbf{A}_{k} s_{k}+\mathbf{B}_{k} s_{k}^{*}\right),
$$

where $\mathbf{s}_{k}(k=1,2, \ldots, K)$ denotes information symbols selected from an alphabet to be transmitted within the $T$ time slots, and $\mathbf{A}_{k}$ and $\mathbf{B}_{k}$ denote the $M \times N$ matrices. Let $\mathbf{s}=\left[s_{1}, s_{2}, \ldots, s_{k}\right]^{T}$. At the receiver, the received signal can be written as 


$$
\mathbf{R}=\sqrt{\frac{\rho}{M}} \mathbf{H S}+\mathbf{V},
$$

where $\rho$ is the signal-to-noise ratio per receiver antenna, $\mathbf{R}=\left[\mathbf{r}_{1}, \mathbf{r}_{2}, \ldots, \mathbf{r}_{T}\right]$, and $\mathbf{V}=\left[\mathbf{v}_{1}, \mathbf{v}_{2}, \ldots, \mathbf{v}_{T}\right]$ denote the $N \times T$ received signal and noise matrices, respectively. Here, $\mathbf{H}$ is an $N \times M$ channel matrix. Throughout the paper, we assume that $\mathbf{H}, \mathbf{S}$ and $\mathbf{V}$ are independent and that the channel coefficients $h_{n m}(m=1, \ldots, M, n=1, \ldots, N)$, of the matrix $\mathbf{H}$ are samples of independent circularly symmetric zero-mean complex white Gaussian random variables with unit variances. An equivalent model can be established by vectoring both sides of (2) such that [5]

$$
\operatorname{vec}(\mathbf{R})=\sqrt{\frac{\rho}{M}} \mathcal{H}\left(\mathcal{A} \mathbf{s}+\mathcal{B} \mathbf{s}^{*}\right)+\operatorname{vec}(\mathbf{V}),
$$

where $\mathcal{H}=\mathbf{I}_{T} \otimes \mathbf{H}, \mathcal{A}=\left[\operatorname{vec}\left(\mathbf{A}_{1}\right), \operatorname{vec}\left(\mathbf{A}_{2}\right), \ldots, \operatorname{vec}\left(\mathbf{A}_{K}\right)\right]$ and $\mathcal{B}=\left[\operatorname{vec}\left(\mathbf{B}_{1}\right), \operatorname{vec}\left(\mathbf{B}_{2}\right), \ldots, \operatorname{vec}\left(\mathbf{B}_{K}\right)\right]$. Combining (3) and its conjugate, and applying a transformation matrix $\mathbf{T}$ to the combined equation, it results in a model that involves only real multiple inputs and real multiple outputs such that

$$
\left[\begin{array}{c}
\operatorname{vec}\left(\mathbf{R}_{r e}\right) \\
\operatorname{vec}\left(\mathbf{R}_{i m}\right)
\end{array}\right]=\sqrt{\frac{\rho}{M}} \mathbf{T}^{H} \tilde{\mathcal{H}} \mathcal{F} \mathbf{T}\left[\begin{array}{c}
\mathbf{s}_{r e} \\
\mathbf{s}_{i m}
\end{array}\right]+\left[\begin{array}{c}
\operatorname{vec}\left(\mathbf{V}_{r e}\right) \\
\operatorname{vec}\left(\mathbf{V}_{i m}\right)
\end{array}\right]
$$

where the unitary transformation matrix is given by

$$
\mathbf{T}=\frac{1}{\sqrt{2}}\left[\begin{array}{cc}
I & j I \\
I & -j I
\end{array}\right]
$$

the precoder matrix is given by

$$
\mathcal{F}=\left[\begin{array}{cc}
\mathcal{A} & \mathcal{B} \\
\mathcal{B}^{*} & \mathcal{A}^{*}
\end{array}\right]
$$

the matrix $\tilde{\mathcal{H}}$ is defined as $\tilde{\mathcal{H}}=\operatorname{diag}\left(\mathcal{H}, \mathcal{H}^{*}\right)$, and $[\cdot]_{\text {re }}$ and $[\cdot]_{i m}$ denote the real and imaginary parts of a quantity, respectively. Eq. (4) is useful in analyzing the detection performance of an LD code at the receiver as well as the ergodic capacity of the LD coded channel.

\section{DESIGN OF LD CODES}

\section{A. Design of FR-FD LD Codes}

In this subsection, we examine the structure of an LD code used in MIMO communications and give the design of FR-FD LD codes proposed in [5].

Firstly, from an information-theoretic viewpoint, we found that a good LD code should have an interunitary structure. To obtain a capacity optimum coding matrix $\mathcal{F}$ such that it renders the ergodic capacity of the LD coded channel equal to that of the original uncoded channel, we have the following assertion [7].

Theorem 1: Let $K=M T$ (i.e., consider full symbol rate transmission). Then, subject to the power constraint:

$$
\sum_{k=1}^{K} \operatorname{tr}\left(\mathbf{A}_{k} \mathbf{A}_{k}^{H}+\mathbf{B}_{k} \mathbf{B}_{k}^{H}\right)=M T,
$$

$\mathcal{F}$ is capacity-optimal if and only if $\mathcal{F}$ is unitary, or equivalently, the following conditions hold:

$$
\begin{gathered}
\operatorname{tr}\left(\mathbf{A}_{k} \mathbf{A}_{k^{\prime}}^{H}+\mathbf{B}_{k^{\prime}} \mathbf{B}_{k}^{H}\right)=\delta\left(k-k^{\prime}\right), \\
\operatorname{tr}\left(\mathbf{B}_{k} \mathbf{A}_{k^{\prime}}^{H}+\mathbf{B}_{k^{\prime}} \mathbf{A}_{k}^{H}\right)=0,
\end{gathered}
$$

for $k, k^{\prime}=1,2, \ldots, K$, where $\delta\left(k-k^{\prime}\right)$ is the Kronecker delta.

Secondly, from a detection error viewpoint, a good LD code should have an intraunitary structure. In the following, we explore the properties of a good code by establishing the lower bound of the worst case pairwise error probability (PEP) and examining the necessary code structure for the lower bound to be reached.

Let us first define the minimum distance of the constellation $\mathcal{S}$ as $d_{\min }(\mathcal{S})=\min _{s, s^{\prime} \in \mathcal{S}^{K}, s \neq s^{\prime}}\left|s-s^{\prime}\right|$. The following theorem provides us with an expression on the universal lower bound of the worst case PEP for any linear dispersion code [8].

Theorem 2: Let $\mathcal{S}_{r e}$ and $\mathcal{S}_{i m}$ denote two constellations consisting of, respectively, the real and the imaginary parts of the elements in constellation $\mathcal{S}$. If $\mathcal{S}$ satisfies the following geometrical property:

$$
d_{\text {min }}(\mathcal{S})=d_{\text {min }}\left(\mathcal{S}_{r e}\right)=d_{\text {min }}\left(\mathcal{S}_{i m}\right),
$$

then for any LD code $\mathcal{F}$ with a power budget $\operatorname{tr}\left(\mathcal{F F}^{H}\right) \leq$ $M T$, the lower bound of the worst case PEP of the ML detector is given by

$$
\max _{s, s^{\prime} \in \mathcal{S}^{K}, s \neq s^{\prime}} P_{\mathcal{F}}\left(s \rightarrow s^{\prime}\right) \geq J\left(\frac{\rho T d_{\min }^{2}(\mathcal{S})}{8 M K}\right),
$$

where $J(a)=(1 / \pi) \int_{0}^{\pi / 2}\left(1+a / \sin ^{2}(\theta)\right)^{-M N} d \theta$ for $a>0$. Furthermore, a necessary condition for the lower bound to be achieved is that the pair of matrices, $\mathbf{A}_{k}$ and $\mathbf{B}_{k}$, associated with each individual symbol must satisfy the following conditions:

$$
\mathbf{A}_{k} \mathbf{A}_{k}^{H}+\mathbf{B}_{k} \mathbf{B}_{k}^{H}=\frac{T}{K} \mathbf{I}_{M}, \mathbf{A}_{k} \mathbf{B}_{k}^{H}+\mathbf{B}_{k} \mathbf{A}_{k}^{H}=0,
$$

Theorem 2 establishes from a detection viewpoint that each individual code matrix of a good LD code should have an intraunitary structure. The following comments on the result should be noted.

1) For the lower bound of (11) to be valid, the constellation $\mathcal{S}$ has to satisfy (10). For example, the square q-ary QAM satisfies the condition, whereas nonsquare q-ary QAM and q-ary PSK do not.

2) The conditions in (12) is necessary but not sufficient.

Then we should strive for both interunitary and intraunitary structures in the design of an LD code, and call such structures of an LD code trace orthonormality [8], which can be formally defined as follows.

Definition 1: Let $T \geq M$. A sequence of $M \times T$ matrices $\mathbf{A}_{k}$ and $\mathbf{B}_{k}, k=1,2, \ldots, K$, and $K \leq M T$ is said to constitute a trace-orthonormal LD code if the following conditions are satisfied: 


$$
\begin{gathered}
\mathbf{A}_{k} \mathbf{A}_{k}^{H}+\mathbf{B}_{k} \mathbf{B}_{k}^{H}=\frac{T}{K} \mathbf{I}_{M}, \\
\mathbf{A}_{k} \mathbf{B}_{k}^{H}+\mathbf{B}_{k} \mathbf{A}_{k}^{H}=0, \\
\operatorname{tr}\left(\mathbf{A}_{k} \mathbf{A}_{k^{\prime}}^{H}+\mathbf{B}_{k^{\prime}} \mathbf{B}_{k}^{H}\right)=\frac{M T}{K} \delta\left(k-k^{\prime}\right), \\
\operatorname{tr}\left(\mathbf{B}_{k} \mathbf{A}_{k^{\prime}}^{H}+\mathbf{B}_{k^{\prime}} \mathbf{A}_{k}^{H}\right)=0,
\end{gathered}
$$

for $k, k^{\prime}=1,2, \ldots, K$. In particular, when $K=M T$, it is said to constitute a trace-orthonormal LD code of full transmission symbol rate.

Now we develop a scheme for the construction of traceorthonormal LD codes. First, we introduce the following definition and then present the systematic generation of traceorthonormal LD codes [5].

Definition 2: Let $T=L M$, where $L$ is a positive integer. A $2 T \times 2 T$ unitary matrix $\mathrm{V}$ satisfying

$$
\mathbf{V}=\left[\begin{array}{cc}
\mathbf{X} & \mathbf{Y} \\
\mathbf{Y}^{*} & \mathbf{X}^{*}
\end{array}\right]
$$

is said to be of $\mathrm{V}$-structure if the following exists:

a) the entries of the $T \times T$ matrices $\mathrm{X}$ and $\mathrm{Y}$ satisfy

$$
\sum_{n=0}^{L-1}\left|x_{(M n+m), t}\right|^{2}+\sum_{n=0}^{L-1}\left|y_{(M n+m), t}\right|^{2}=\frac{1}{M},
$$

for $t=1,2, \ldots T$; and $m=1,2, \ldots M$;

b) the cross terms between $\mathrm{X}$ and Y satisfy

$$
\sum_{n=0}^{L-1}\left(x_{(M n+m), t} y_{(M n+m), t}^{*}+x_{(M n+m), t}^{*} y_{(M n+m), t}\right)=0,
$$

for $t=1,2, \ldots T$; and $m=1,2, \ldots M$.

Theorem 3: Let $T=L M$, and $K=R T$ with $R \leq M$. Suppose that we have the V-structured $2 T \times 2 T$ matrices $\mathbf{V}_{r}$, for $r=1, \ldots, R$, respectively given by (17). By taking the $t$ th column vectors of the component matrices $\mathbf{X}_{r}, \mathbf{Y}_{r}$ of $\mathbf{V}_{r}$, we now form the matrices $\mathbf{D}_{t}$ and $\Delta_{t}$ as follows:

$$
\begin{gathered}
\mathbf{D}_{t}=\left[\operatorname{diag}\left(x_{1, t}, \ldots x_{M, t}\right), \operatorname{diag}\left(x_{M+1, t}, \ldots x_{2 M, t}\right), \cdots\right. \\
\left.\operatorname{diag}\left(x_{(L-1) M+1, t}, \ldots x_{L M, t}\right)\right] \\
\Delta_{t}=\left[\operatorname{diag}\left(y_{1, t}, \ldots y_{M, t}\right), \operatorname{diag}\left(y_{M+1, t}, \ldots y_{2 M, t}\right), \ldots\right. \\
\left.\operatorname{diag}\left(y_{(L-1) M+1, t}, \ldots y_{L M, t}\right)\right]
\end{gathered}
$$

for $r=1, \ldots R$ and $t=1, \ldots T$. Let two sequences of matrices $\mathbf{A}_{r, t}$ and $\mathbf{B}_{r, t}$ be given by

$$
\mathbf{A}_{r, t}=\sqrt{\frac{M T}{K}} \mathbf{C}_{M}^{r-1} \mathbf{D}_{t}, \mathbf{B}_{r, t}=\sqrt{\frac{M T}{K}} \mathbf{C}_{M}^{r-1} \Delta_{t},
$$

where $\mathbf{C}_{M}=\left[\begin{array}{cc}\mathbf{O}_{1 \times(P-1)} & 1 \\ \mathbf{I}_{P-1} & \mathbf{O}_{(P-1) \times 1}\end{array}\right]$. Then the matrix family $\mathbf{A}_{r, t}, \mathbf{B}_{r, t}$ constitutes a trace-orthonormal LD code with a symbol rate $R$ per channel use.
We now show how to select $\mathrm{V}$-structured matrices from the trace-orthonormal LD code family such that the resulting codes will provide full diversity. Meanwhile we state the main result for the design of trace-orthonormal full diversity LD codes as follows [5].

Theorem 4: 4: Let $K=R T$ with $T=M \widetilde{L}$ and $0<R \leq$ $M$. Also, let $M=\prod_{m=1}^{d} q_{m}^{\lambda_{m}} \prod_{k=1}^{d_{M}} p_{k}^{\alpha_{k}}, R=\prod_{m=1}^{d} q_{m}^{\overline{\mu_{i}}}$ $\prod_{l=1}^{d_{R}} \widetilde{p}_{l}^{\beta_{l}}$ and $L=L_{0} \prod_{i=1}^{d} q_{m}^{\tau_{m}} \prod_{k=1}^{\bar{d}_{M}} p_{k}^{\gamma_{k}} \prod_{l=1}^{d_{R}} \widetilde{p}_{l}^{\rho_{l}}$, where $q_{m}, p_{k}$ and $\widetilde{p}_{l}$ are primes, $\alpha_{k}, \beta_{l}, \gamma_{i}, \rho_{l} \geq 1$, and $L_{0}$ is prime to both $M$ and $R$. We choose the $\mathrm{V}$-structured matrices in Theorem 3 as follows:

$$
\begin{gathered}
X_{r}=\zeta_{L R M^{2}}^{r-1} \mathbf{U} \otimes\left[\begin{array}{c}
\left.\mathcal{R}_{0}\left[1: M_{1},:\right]\right], \\
\mathbf{O}_{M_{2} \times M}
\end{array}\right], \\
Y_{r}=\zeta_{L R M^{2}}^{r-1} \mathbf{U} \otimes\left[\begin{array}{c}
\mathbf{O}_{M_{1} \times M} \\
\mathcal{R}_{0}^{*}\left[M_{1}+1: M,:\right]
\end{array}\right],
\end{gathered}
$$

for $r=1,2, \ldots R$, where $M_{1}+M_{2}=M, M_{1}, M_{2} \geq 0$, $\mathbf{U}$ is an arbitrarily given $\widetilde{L} \times \widetilde{L}$ unitary matrix and $\mathcal{R}_{0}=$ $\mathbf{W}_{M}^{H} \operatorname{diag}\left(1, \zeta_{P}, \ldots \zeta_{P}^{M-1}\right)$. Then the resulting signal matrix $\mathbf{S}$ provides full diversity over any constellation carved from $\mathbb{Z}^{K}\left[\zeta_{L}\right]$ with a symbol transmission rate $R$ symbols per channel use.

\section{B. Coordinate-Interleaving LD Codes (CILDC)}

In [6], the authours propose a new LD codes encoding procedure using coordinate interleaving as follows: Consider a pair of source data symbol vectors $\mathbf{s}^{1}=\left[s_{1}^{1}, s_{2}^{1}, \ldots, s_{K}^{1}\right]^{T}$ and $\mathbf{s}^{2}=\left[s_{1}^{2}, s_{2}^{2}, \ldots, s_{K}^{2}\right]^{T}$, where $s_{k}^{i}=\operatorname{Re}\left(s_{k}^{i}\right)+j \operatorname{Im}\left(s_{k}^{i}\right)$ for $i=1,2$ and $k=1, \ldots, K$. The transmitter first coordinateinterleaves $\mathbf{s}^{1}$ and $\mathbf{s}^{2}$ into $\mathbf{s}^{C I 1}=\left[s_{1}^{C I 1}, s_{2}^{C I 1}, \ldots, s_{K}^{C I 1}\right]^{T}$ and $\mathbf{s}^{C I 2}=\left[s_{1}^{C I 2}, s_{2}^{C I 2}, \ldots, s_{K}^{C I 2}\right]^{T}$, where $s_{k}^{C I 1}=\operatorname{Re}\left(s_{k}^{1}\right)+$ $j \operatorname{Im}\left(s_{k}^{2}\right)$ and $s_{k}^{C I 2}=\operatorname{Re}\left(s_{k}^{2}\right)+j \operatorname{Im}\left(s_{k}^{1}\right)$. Then, $\mathbf{s}^{C I 1}$ and $\mathbf{s}^{C I 2}$ are encoded into two LD codewords of size $T \times M$, $\mathbf{S}^{C I 1}$ and $\mathbf{S}^{C I 2}$, respectively. Then the transmitter successively sends $\mathbf{S}^{C I 1}$ and $\mathbf{S}^{C I 2}$ during two interleaved periods.

$\mathrm{Su}$ and Liu [9] recently analyzed the diversity of spacetime modulation over Rayleigh fading channels. A modified strategy can be used to investigate the diversity of CILDC systems [6].

Consider a CILDC block $C$, which consists of two LD codewords of size $T \times M, \mathbf{S}^{C I i}$, where $i=1,2$. The communication model for the CILDC block $C$ can be rewritten as

$$
\overline{\mathbf{R}}=\sqrt{\frac{\rho}{M}} \overline{\mathbf{S H}}+\overline{\mathbf{V}}
$$

where the noise vector is $\overline{\mathbf{V}}$; the received signal vector $\overline{\mathbf{R}}=$ $\left[\left[\overline{\mathbf{R}}^{1}\right]^{T},\left[\overline{\mathbf{R}}^{2}\right]^{T}\right]^{T}$, where $\overline{\mathbf{R}}^{i}=\left[\operatorname{vec}\left(\mathbf{R}^{i}\right)\right] ; \overline{\mathbf{S}}$ is the channel symbol matrix corresponding to the block $C, \overline{\mathbf{S}}=\operatorname{diag}\left(\overline{\mathbf{S}}^{1}, \overline{\mathbf{S}}^{2}\right)$, where $\overline{\mathbf{S}}^{i}=I_{N} \otimes \operatorname{diag}\left(\overline{\mathbf{S}}_{1}^{i}, \ldots, \overline{\mathbf{S}}_{M}^{i}\right), \overline{\mathbf{S}}_{m}^{i}=\operatorname{diag}\left(\left[\mathbf{S}^{i}\right]_{1, m}, \ldots\right.$, $\left.\left[\mathbf{S}^{i}\right]_{T, m}\right), k=1,2$ and $m=1, \ldots, M$; the channel vector $\overline{\mathbf{H}}=\left[\left[\mathbf{H}^{1}\right]^{T},\left[\mathbf{H}^{2}\right]^{T}\right]^{T}$, where $\mathbf{H}^{i}=\left[\left(\mathbf{h}_{1,1}^{i}\right)^{T}, \ldots,\left(\mathbf{h}_{M, 1}^{i}\right)^{T}, \ldots\right.$, $\left.\left(\mathbf{h}_{1, N}^{i}\right)^{T}, \ldots,\left(\mathbf{h}_{M, N}^{i}\right)^{T}\right]^{T}$, and $\mathbf{h}_{m, n}^{i}=\left[h_{m, n}^{k, 1}, \ldots, h_{m, n}^{k, T}\right]$. 
Consider the directed pair of matrices $\overline{\mathbf{S}}$ and $\widetilde{\overline{\mathbf{S}}}$ corresponding to two different blocks $C$ and $\widetilde{C}$. The upper bound pairwise error probability [10] is

$$
P(\overline{\mathbf{S}} \rightarrow \widetilde{\mathbf{S}}) \leq\left(\begin{array}{c}
2 r-1 \\
r
\end{array}\right)\left(\prod_{a=1}^{r} \gamma_{a}\right)^{-1}\left(\frac{\rho}{M}\right)^{-r},
$$

where $r$ is the rank of $(\overline{\mathbf{S}}-\widetilde{\overline{\mathbf{S}}}) \mathbf{R}_{H}(\overline{\mathbf{S}}-\widetilde{\overline{\mathbf{S}}})^{H}$, and $\mathbf{R}_{H}=$ $E\left[\overline{\mathbf{H}}[\overline{\mathbf{H}}]^{H}\right]$ is the correlation matrix of vector $\overline{\mathbf{H}}, a=1, \ldots, r$ are the non-zero eigenvalues of $\Lambda=(\overline{\mathbf{S}}-\widetilde{\overline{\mathbf{S}}}) \mathbf{R}_{H}(\overline{\mathbf{S}}-\widetilde{\overline{\mathbf{S}}})^{H}$. Then the rank and product criteria are

1) Rank criterion: the minimum rank of $\Lambda$ over all direction pairs of different matrices $\overline{\mathbf{S}}$ and $\widetilde{\mathbf{S}}$ should be as large as possible.

2) Product criterion: the minimum value of the product $\prod_{a=1}^{r} \gamma_{a}$ over all direction pairs of different $\overline{\mathbf{S}}$ and $\widetilde{\mathbf{S}}$ should be maximized.

To maximize the rank of $\Lambda$, we need to maximize the ranks of both $\mathbf{R}_{H}$ and $\overline{\mathbf{S}}-\widetilde{\overline{\mathbf{S}}}$. Denote

$$
\Omega^{k}=\overline{\mathbf{S}}^{k}-\widetilde{\mathbf{S}^{k}}
$$

where $k=1,2$.

Assume that all the possible $\overline{\mathbf{S}}^{k}$ and $\widetilde{\overline{\mathbf{S}}^{k}}$ are contained in a set $\mathcal{S}^{k}$,i.e. $\overline{\mathbf{S}}^{k}, \widetilde{\overline{\mathbf{S}}^{k}} \in \mathcal{S}^{k}$, where $k=1,2$. Then the diversity order of the CILDC, $r_{d}$ is

$$
r_{d}=\min \{\operatorname{rank}(\Lambda), \overline{\mathbf{S}}, \widetilde{\overline{\mathbf{S}}} \in \mathcal{S}, \overline{\mathbf{S}} \neq \widetilde{\overline{\mathbf{S}}}\} .
$$

When $\overline{\mathbf{S}} \neq \widetilde{\mathbf{S}}$, there are three distinct categories of situations, 1) $\overline{\mathbf{S}}^{1} \neq \widetilde{\mathbf{S}}^{1}$ and $\overline{\mathbf{S}}^{2}=\widetilde{\widetilde{\mathbf{S}}^{2}}$,

2) $\overline{\mathbf{S}}^{1}=\widetilde{\widetilde{\mathbf{S}}^{1}}$ and $\overline{\mathbf{S}}^{2} \neq{\widetilde{\overline{\mathbf{S}}^{2}}}_{\widetilde{\mathbf{S}}^{2}}$,

3) $\overline{\mathbf{S}}^{1} \neq \widetilde{\mathbf{\mathbf { S }}^{1}}$ and $\overline{\mathbf{S}}^{2} \neq \widetilde{\overline{\mathbf{S}}^{2}}$.

Note that when $\mathbf{R}_{H}$ is full rank,

1) in the above situations (1) and (2), the upper bound of $\operatorname{rank}(\Lambda)$ is NT;

2) in the above situation (3), the upper bound of $\operatorname{rank}(\Lambda)$ is $2 \mathrm{NT}$.

Thus CILDC does increase $r$ for the above-mentioned third situation, which is not the conventional diversity order of the LD codes and may significantly impact system performance. It is necessary to introduce a new concept to quantify this effect as follows.

Definition 3: Statistical diversity order, $r_{s d}$ is the rank of $\Lambda$ achieved with a certain probability $\alpha$, mathematically written as

$$
P\left\{\operatorname{rank}(\Lambda) \geq r_{s d}, \overline{\mathbf{S}} \neq \widetilde{\overline{\mathbf{S}}},\{\overline{\mathbf{S}}, \widetilde{\mathbf{S}}\} \in \mathcal{S}\right\}=\alpha,
$$

Then we have the following theorem [6].

Theorem 5: A CILDC is constructed through coordinate interleaving across a pair of component LDC codewords. Both component LDC encoders are able to generate different codewords for different input sequences. The diversity orders of the component LDCs are $r_{d}^{1}$ and $r_{d}^{2}$, respectively. Suppose that $\mathbf{R}_{H}$ is full rank. The codebook sizes of the two component LDCs are the same value $N_{a}$.

1) The diversity order of this CILDC, $r_{d}$ is $\min \left\{r_{d}^{1}, r_{d}^{2}\right\}$.

2) Assuming that all directional pairs $\overline{\mathbf{S}}$ and $\widetilde{\overline{\mathbf{S}}}$ are equally probable, the statistical diversity order of this CILDC, $r_{s d}$ is $r_{d}^{1}+r_{d}^{2}$ with probability

$$
\alpha=\frac{\left(\begin{array}{c}
N_{a} \\
2
\end{array}\right)\left(\begin{array}{c}
N_{a} \\
2
\end{array}\right)}{\left(\begin{array}{c}
N_{a} \\
2
\end{array}\right)\left(\begin{array}{c}
N_{a} \\
2
\end{array}\right)+N_{a}\left(\begin{array}{c}
N_{a} \\
2
\end{array}\right)},
$$

As mentioned in Theorem 2, for FR-FD LD codes, the constellation $\mathcal{S}$ has to satisfy (10). This restricts the constellations that we can use, such as q-ary PSK, nonsqure qQAM. Now, the method presented above can help us. Using coordinate interleaving we can improve the performance with some constellations, which do not satisfy the condition, as we can see in the simulation in both block and fast fading channels.

\section{SIMULATION}

In this section, we give the examples to show the performance of our method. In the procedure, perfect channel knowledge is assumed at the receiver but not at the transmitter. Channel symbols are estimated using MMSE estimation. The codes presented in [5] are used as component LDC coding matrices of CILDC systems in the simulation, and 4-PSK, 8PSK, nonsquare 8-QAM is used since they do not satisfy the condition in (10).

The performance comparison of the code given in example $3(M=2, N=2, K=4, T=2)$ in [5] is shown in Figure 1 and 3, with 4-PSK and 8-PSK, respectively. The performance comparison of the code given in example $4(M=3, N=3$, $K=9, T=3$ ) in [5] is shown in Figure 2 and 4, with 4PSK and 8-QAM, respectively. All these codes are simulated in block and fast fading channels.

Although these constellations do not satisfy the condition in (10), as shown in these figures, using coordinate interleaving, the performance are improved at high SNRs in both channels.

\section{CONCLUSION}

In this paper, we improve the Trace-orthonormal fulldiversity cyclomic space-time codes using coordinate interleaving procedure, which enables not only symbol-level diversity but also coordinate-level diversity for high rate $\mathrm{LD}$ code design. Although the constellations is restricted in the design of full-rate full-diversity linear dispersion codes (FRFD LDC), CILDC show either much higher average diversity order or extra coding advantage in both block and fast fading channels at high SNRs with the constellations which do not satisfy the restriction as presented in the simulation. 


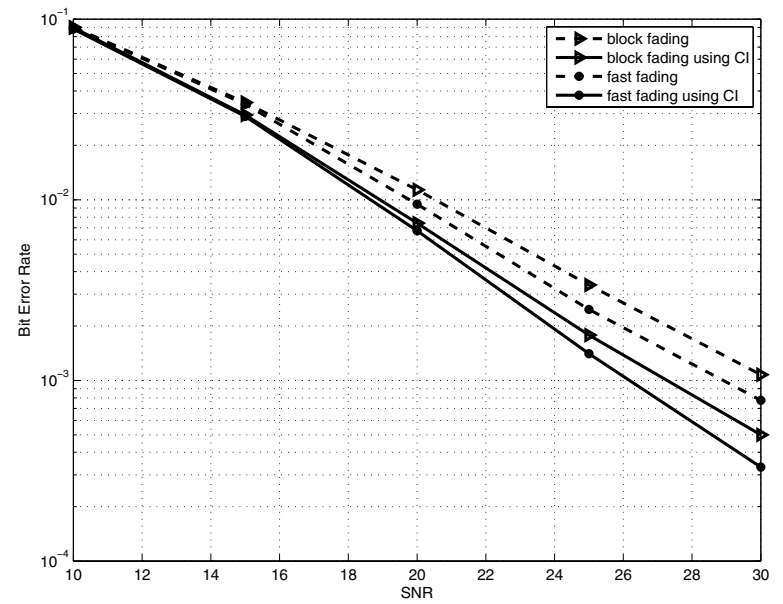

Fig. 1. BER performance comparison of LDC vs CILDC: $M=2, N=$ $2, K=4, T=2$ with 4-PSK

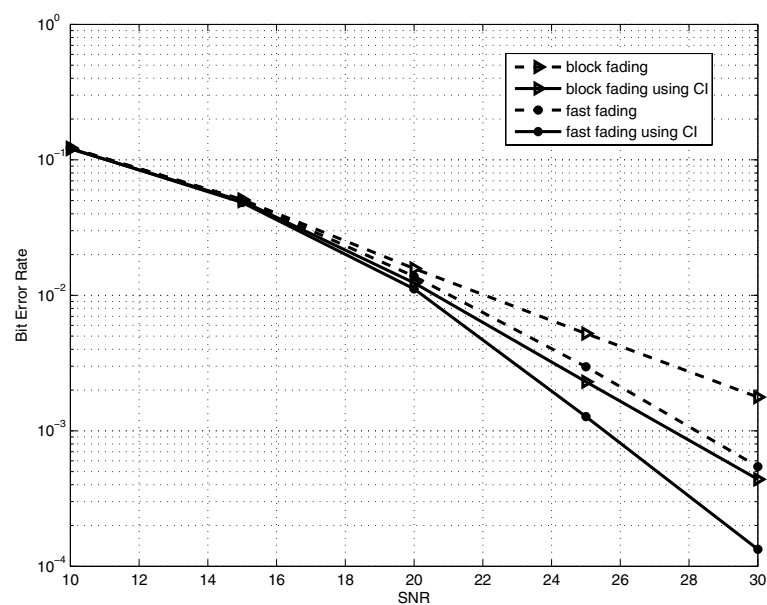

Fig. 2. BER performance comparison of LDC vs CILDC: $M=3, N=$ $3, K=9, T=3$ with 4-PSK

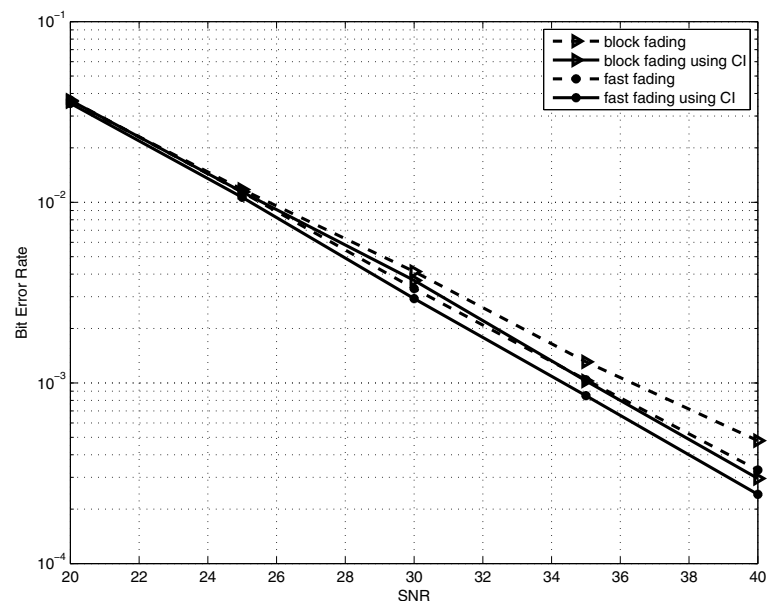

Fig. 3. BER performance comparison of LDC vs CILDC: $M=2, N=$ $2, K=4, T=2$ with 8 -PSK

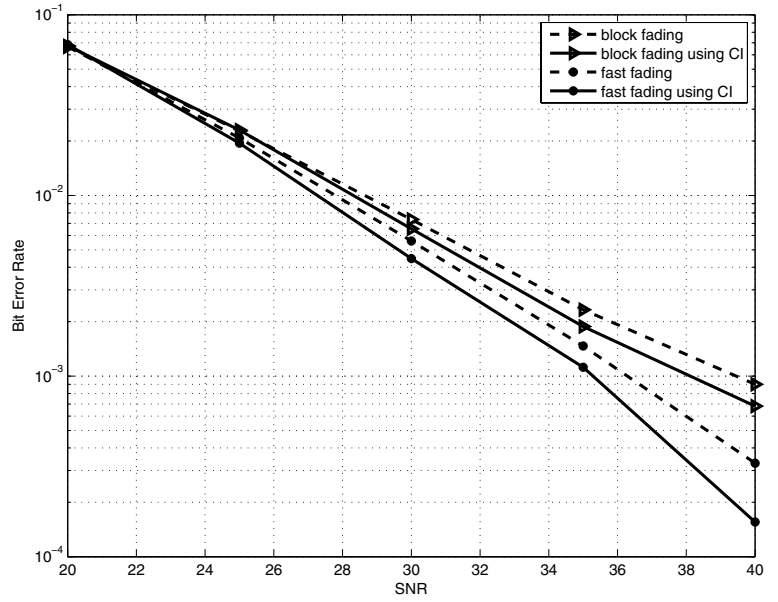

Fig. 4. BER performance comparison of LDC vs CILDC: $M=3, N=$ $3, K=9, T=3$ with 8 -QAM

\section{ACKNOWLEDGMENT}

This work is supported by NSF China \#60672067, by NSF Shanghai \#06ZR14041, by Shanghai-Canada NRC \#06SN07112, by Cultivation Fund of the Key Scientific and Technical Innovation Project, Ministry of Education of China \#706022, by Program for New Century Excellent Talents in University \#NCET-06-0386, and by PUJIANG Talents \#07PJ4046.

\section{REFERENCES}

[1] I. Teletar, "Capacity of multi-antenna gaussian channels," Euro. Trans.Telecomm., vol. 10, pp. 585-595, Nov. 1999.

[2] G. Foschini, M. Gans, "On limits of wireless communications in fading envioronments when using multiple antennas," Wireless Personal Commun., vol. 6, pp. 311-335,Mar. 1998.

[3] B. Hassibi, B. Hochwald, "High-rate codes that are linear in space and time," IEEE Trans. Inform. Theory, vol. 48, no. 7, pp. 1804-1824, Jul. 2002.

[4] R. W. Heath and A. J. Paulraj "Linear dispersion codes for MIMO systems based on frame theory," IEEE Trans. Signal Process, vol. 50, no. 10 pp. 2429-2441, Oct. 2002.

[5] J. K. Zhang, J. Liu, and K. M. Wong, "Trace-orthonormal full-diversity cyclotomic space-time codes," IEEE Trans. Signal Process, vol. 55, no. 2 pp. 618-630, Feb. 2007.

[6] J. S. Wu and S. D. Blostein, "Space-time linear dispersion using coordinate interleaving," ISIT 2006, Seattle, USA, pp. 386-390, Jul. 2006.

[7] J. Liu, "Optimal linear STBC Design for MIMO systems with MMSE receivers," M.A.Sc. thesis, Electrical and Computer Eng. Dept., McMaster University, Hamilton, ON, Canada 2004.

[8] J. K. Zhang, J. Liu, and K. M. Wong,“Trace-orthonormal full-diversity triangular cyclotomic space-time codes minimizng worst case pair-wise error probability," Int. Conf. Sensor Array Multichannel Signal Processing, Sitges, Barcelona, Spain, Jul. 2004.

[9] W. Su, Z. Safar and K. J. R. Liu,"Diversity analysis of space-time modulation over time-correlated Rayleigh-fading channels," IEEE Trans. Inform. Theory, vol. 50, no. 8, pp. 1832-1840, Aug. 2004.

[10] S. Siwamogsatham, M. P. Fitz, and J. H. Grimm, "A new view of perpormance analysis of transmit diversity schemes in correlated Rayleigh fading," IEEE Trans. Inform. Theory, vol. 48, no. 4, pp. 950-956, Apr. 2002. 